\title{
Semi-active suspension with semi-active inerter and semi-active damper ${ }^{\star}$
}

\author{
Michael Z. Q. Chen*, Yinlong Hu ${ }^{* *}$, Chanying Li ${ }^{* * *}$, and \\ Guanrong Chen ${ }^{* * * *}$ \\ * Department of Mechanical Engineering, The University of Hong \\ Kong, Hong Kong. \\ ** School of Automation, Nanjing University of Science and \\ Technology, Nanjing, China. Email: yinlong.h@gmail.com. \\ *** Academy of Mathematics and Systems Sciences, Chinese Academy \\ of Science, Beijing, China. \\ **** Department of Electronic Engineering, City University of Hong \\ Kong, Hong Kong.
}

\begin{abstract}
This paper investigates the application of semi-active inerter in semi-active suspension. A semi-active inerter is defined as an inerter whose inertance can be adjusted within a finite bandwidth by on-line control actions. A force-tracking approach to designing semi-active suspension with a semi-active inerter and a semi-active damper is proposed, where the target active control force derived by LQR control in the "Reciprocal State-Space" (RSS) framework is tracked by controlling the semi-active damping coefficient and semi-active inertance. One of the advantages of the proposed method is that it is straightforward to use the acceleration information in the controller design. Simulation results demonstrate that the semi-active suspension with a semi-active inerter and a semi-active damper can track the target active control force much better than the conventional semi-active suspension (which only contains a semi-active damper) does. As a consequence, the overall performance in ride comfort, suspension deflection and road holding is improved, which effectively demonstrates the necessity and the benefit of introducing semi-active inerter in vehicle suspension.
\end{abstract}

Keywords: Inerter, semi-active suspension, Reciprocal State-Space.

\section{INTRODUCTION}

In vehicle suspension, several conflicting purposes are served, where the most basic one is to isolate the vehicle body from road irregularities within a small suspension deflection while maintaining the tyres adhering to the road permanently (Gao, Sun, \& Shi (2010); Gao, Lam, \& Wang (2006); Du, Sze, \& Lam (2005)). The ability of passive suspensions to handle such a conflict is limited since the passive element coefficients (spring stiffness, damping coefficient and inertance (Smith (2002)) ) cannot be adjusted by on-line control actions. In contrast, active suspensions can improve the overall performance simultaneously (Du \& Zhang $(2007,2009))$. However, the high level of energy consumption prevents it from being extensively used in practice. Semi-active suspension achieves a good comprise between the hight cost of energy consumption and the performances by making the spring stiffness and the damping coefficient controllable (Du, Sze, \& Lam (2005)). For more details of these three types of suspension, see (Gao, Sun, \& Shi (2010); Gao, Lam, \& Wang (2006); Du, Sze, \& Lam (2005); Du \& Zhang (2007, 2009); Li et al. (2012)) and references therein.

\footnotetext{
^ This research was partially supported by the National Natural Science Foundation of China under Grants 61374053 and 61203067 and the Hong Kong University Committee on Research and Conference Grants under Grant 201111159110.
}

The concept of inerter is introduced in (Smith (2002)), which is defined as a mechanical two-terminal device with the property that the equal and oppose forces applied at its two terminals are proportional to the relative acceleration between them (Smith (2002); Chen et al. (2009)). The applications of inerter in various mechanical structures have been investigated (Smith \& Wang (2004); Chen, Hu, \& Du (2012); Chen et al. (2014)), where the benefits of employing inerter have been well demonstrated. The interest in passive network synthesis has also been rekindled (Chen \& Smith (2008, 2009a,b); Wang, \& Chen (2012); Chen et al. (2013); Wang, Chen, \& Hu (2014)).

Note that for all the applications of inerter so far, the inerter is applied as a passive element, in the sense that, the inertance cannot be adjusted by on-line control actions. Considering the significant improvements of employing controllable dampers in vehicle suspension, in this paper we will investigate the benefits of introducing a controllable inerter in vehicle suspension. Here, the controllable inerter or semi-active inerter is defined as an inerter whose inertance can be adjusted within a finite bandwidth.

The semi-active inerter can be viewed as a mechanical component just like the passive inerter in (Smith (2002)), and its realization is only one part of the research on it. However, another equally important aspect is to demonstrate the necessity of introducing a semi-active inerter 
in the first place, which is the main focus of this paper. A force-tracking strategy in designing semi-active suspension with a semi-active inerter and a semi-active damper is proposed. This force-tracking strategy consists of two parts: a target active control law is first provided and then tracked on-line by controlling the coefficients of the employed semi-active inerter and the semi-active damper. To directly use the acceleration information, the target active control law is designed based on the statederivative feedback control approach in the "Reciprocal State-Space" (RSS) framework (Tseng (1997)).

The organization of this paper is as follows. In Section 2, the optimal solution to a general performance index for LQR control in the RSS framework is derived. In Section 3, the force-tracking strategy for semi-active suspension is illustrated based on a quarter-car model. Numerical simulations are performed in Section 4. Conclusions are drawn in Section 5.

\section{RSS FRAMEWORK AND STATE-DERIVATIVE FEEDBACK CONTROL}

\subsection{Overview of state-derivative feedback control}

Traditionally, for a vibration system, the State-Space (SS) representation can be obtained as

$$
\dot{x}=A x+B u,
$$

where $x$ is the state variable and $u$ is the control input vector. If full state feedback control is employed, the control input $u$ can be written as

$$
u=-K x \text {. }
$$

Note that only displacement and velocity information is contained in the state variable $x$. To directly implement acceleration information, it is straightforward to use the state-derivative $\dot{x}$, where acceleration information is contained in the feedback controller design. Therefore, the RSS framework introduced in (Tseng (1997); Kwak, Washington, \& Yedavalli (2002); Duan, Ni, \& Ko (2005)) can be employed as follows:

$$
\begin{aligned}
& x=G \dot{x}+H u, \\
& u=-K \dot{x},
\end{aligned}
$$

where $G=A^{-1}$ and $H=-A^{-1} B$.

2.2 LQR control in the RSS framework with a general performance index

In the SS framework, the general performance index in LQR control can be defined as

$$
J=\int_{0}^{\infty}\left(x^{T} Q^{\prime} x+2 x^{T} N^{\prime} u+u^{T} R^{\prime} u\right) d t,
$$

where $Q^{\prime}, N^{\prime}$, and $R^{\prime}$ satisfy the general assumptions in LQR control (Zhou, Doyle, \& Glover (1996)). Substituting (3) into (5), one can obtain the equivalent index in RSS framework as

$$
J=\int_{0}^{\infty}\left(\dot{x}^{T} Q \dot{x}+2 \dot{x}^{T} N u+u^{T} R u\right) d t
$$

where

$$
\left.\begin{array}{l}
Q=G^{T} Q^{\prime} G, \\
N=G^{T} Q^{\prime} H+G^{T} N^{\prime}, \\
R=H^{T} Q^{\prime} H+2 H^{T} N^{\prime}+R^{\prime} .
\end{array}\right\}
$$

Note that in the existing works about LQR control in RSS framework (Kwak, Washington, \& Yedavalli (2002); Duan, $\mathrm{Ni}, \& \mathrm{Ko}(2005))$, the performance index is always defined in a nonstandard form without the cross product term as

$$
J=\frac{1}{2} \int_{0}^{\infty}\left(\dot{x}^{T} Q \dot{x}+u^{T} R u\right) d t
$$

In this paper, the general performance index (6) will be adopted and the optimal solution is derived as follows:

Theorem 1. Consider the performance index (6) in the RSS framework (3). The full-state-derivative feedback gain matrix $K$ in (4) that minimizes (6) can be obtained as

$$
K=R^{-1}\left(H^{T} P+N^{T}\right),
$$

where $P$ is solved from

$$
\begin{gathered}
\left(G-H R^{-1} N^{T}\right)^{T} P+P\left(G-H R^{-1} N^{T}\right)-P H R^{-1} H^{T} P \\
+Q-N R^{-1} N^{T}=0 .
\end{gathered}
$$

Proof. The proof is omitted for brevity.

Remark 1. It is well known that there exists an unique optimal feedback control law $u_{S S}$ to minimize (5) (Zhou, Doyle, \& Glover (1996)):

$$
u_{S S}=-K_{S S} x
$$

Denote the state-derivative feedback control law derived in Theorem 1 in the RSS framework by

$$
u_{R S S}=-K_{R S S} \dot{x}
$$

If the performance indexes (6) and (5) are equivalent, that is, satisfying (7), then the optimal control law is unique, the following equations hold:

$$
\begin{gathered}
u_{S S}=u_{R S S}, \\
K_{R S S}=K_{S S}\left(A-B K_{S S}\right)^{-1}=K_{S S}\left(G-H K_{R S S}\right) .
\end{gathered}
$$

Remark 1 indicates that by considering the general performance index (6), one can derive the equivalent optimal control law in the RSS framework with the one in the SS framework without any loss of generality, while the merit in the RSS framework is preserved that accelerations are directly used for feedback.

\section{SEMI-ACTIVE SUSPENSION BY FORCE-TRACKING STRATEGY}

In this section, a force-tracking methodology is proposed to design semi-active suspension with a semi-active inerter and a semi-active damper in the RSS framework.

\subsection{Quarter-car model}

The equations of motion for the quarter-car model as shown in Fig. 1 are given by

$$
\begin{aligned}
& m_{s} \ddot{z}_{s}=-k_{s}\left(z_{s}-z_{u}\right)-u, \\
& m_{u} \ddot{z}_{u}=k_{s}\left(z_{s}-z_{u}\right)+u-k_{t}\left(z_{u}-z_{r}\right),
\end{aligned}
$$

where $m_{s}$ and $m_{u}$ are the sprung mass and the unsprung mass, respectively, $k_{s}, k_{t}$ are the spring stiffnesses, $z_{s}, z_{u}$, $z_{r}$ are the displacements of the sprung mass, the unsprung mass and the road in the vertical direction, and $u$ is the suspension actuator force, which is equal to $u_{a}$ or $u_{s}$ for active suspension or semi-active suspension, respectively. 

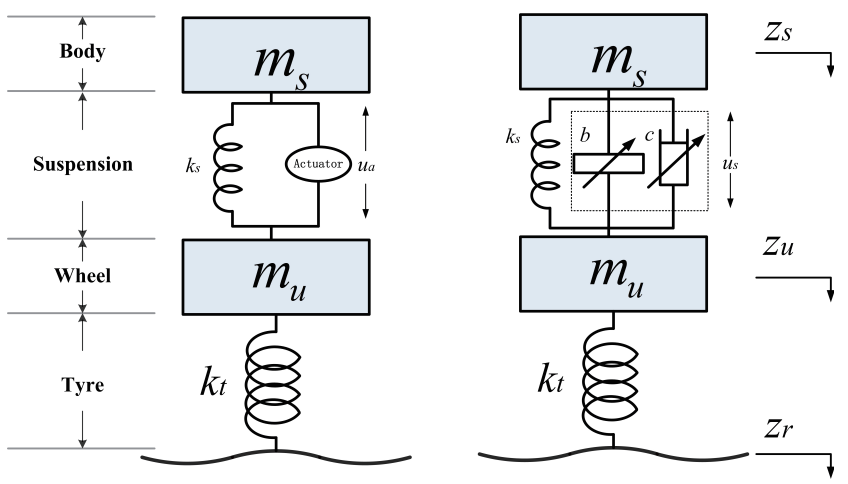

Fig. 1. Quarter-car model: left: active suspension; right: semi-active suspension with a semi-active inerter and a semi-active damper.

In this paper, a first-order actuator model in (Savaresi et al. (2010)) is employed to represent the finite-bandwidth property of the employed actuators, as

$$
\dot{u}=-\beta u+\beta u_{i n},
$$

where the bandwidths of semi-active damper and semiactive inerter are assumed to be identically $30 \mathrm{~Hz}$.

By denoting $x=\left[z_{s}, \dot{z}_{s}, z_{u}, \dot{z}_{u}, u\right]^{T}$, the model in the SS framework can be written as

$$
\dot{x}=A x+B u_{i n}+B_{r} z_{r},
$$

where $B=\left[\begin{array}{lllll}0 & 0 & 0 & 0 & \beta\end{array}\right]^{T}, B_{r}=\left[\begin{array}{lllll}0 & 0 & 0 & \frac{k_{t}}{m_{u}} & 0\end{array}\right]^{T}$, and

$$
A=\left[\begin{array}{ccccc}
0 & 1 & 0 & 0 & 0 \\
-\frac{k_{s}}{m_{s}} & 0 & \frac{k_{s}}{m_{s}} & 0 & -\frac{1}{m_{s}} \\
0 & 0 & 0 & 1 & 0 \\
\frac{k_{s}}{m_{u}} & 0 & -\frac{k_{s}+k_{t}}{m_{u}} & 0 & \frac{1}{m_{u}} \\
0 & 0 & 0 & 0 & -\beta
\end{array}\right]
$$

Then, the model in RSS framework can be obtained as

$$
x=G \dot{x}+H u_{i n}+H_{r} z_{r},
$$

with $G=A^{-1}, H=-A^{-1} B, H_{r}=-A^{-1} B_{r}$. In this paper, the accelerations and suspension force are assumed to be measured. For the quarter-car model, the sprung mass acceleration $\left(\ddot{z}_{s}\right)$, suspension deflection $\left(z_{s}-z_{u}\right)$ and tyre deflection $\left(z_{u}-z_{r}\right)$, representing ride comfort, suspension working space and road holding performance, respectively, are most considered in vehicle suspension design. Hence, the controlled output $z$ is defined as

$$
z=\left[\begin{array}{c}
\ddot{z}_{s} \\
z_{s}-z_{u} \\
z_{u}-z_{r}
\end{array}\right]=C_{z} \dot{x}+D_{z} u_{i n}
$$

with

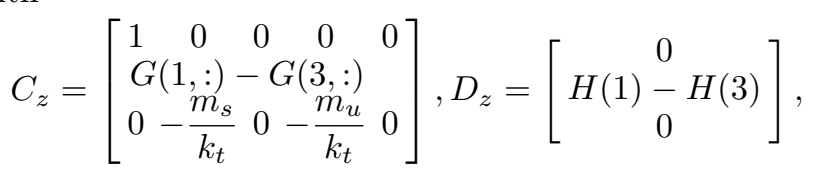

where $G(1,:)$ and $G(3,:)$ denote the first and the third rows of $G$, while $H(1)$ and $H(3)$ denote the first and the third elements of $H$, respectively.

The performance index is defined as

$$
\begin{aligned}
J & =\int_{0}^{\infty} z^{T} Q_{0} z+u_{i n}^{T} r u_{i n} d t \\
& =\int_{0}^{\infty}\left(\dot{x}^{T} Q \dot{x}+2 \dot{x}^{T} N u_{i n}+u_{i n}^{T} R u_{i n}\right) d t
\end{aligned}
$$

where $Q_{0}$ is the weighting matrix determined by the designer defined as $Q_{0}=\operatorname{diag}\left\{\rho_{1}, \rho_{2}, \rho_{3}\right\}$, and $Q=$ $C_{z}^{T} Q_{0} C_{z}, N=C_{z}^{T} Q_{0} D_{z}, R=D_{z}^{T} Q_{0} D_{z}+r$.

\subsection{Target active control law}

In this paper, LQR control in the RSS framework given in Theorem 1 is employed as the target active control law. For the quarter car model shown in (18) and the performance index defined in (20), the target active control law is

$$
u_{a}=-K \dot{x}
$$

where $K$ is solved from (9) and (10).

Since the derivative of suspension force $u$ is unmeasurable, the control law (21) is rewritten as

$$
u_{a}=-K_{1} \dot{\bar{x}}-K_{2} \dot{u},
$$

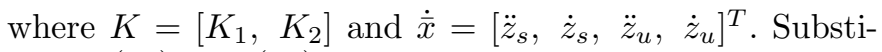
tuting (16) into (22), one obtains

$$
u_{a}=\left(1+K_{2} \beta\right)^{-1}\left(-K_{1} \dot{\bar{x}}+K_{2} \beta u\right) .
$$

\subsection{Force-tracking strategy}

To approximate the target active control law (23), an optimization problem will be solved at each time instant:

$$
\min _{b(t), c(t)}\left|u_{s}(t)-u_{a}(t)\right|
$$

subject to $b_{\min } \leq b(t) \leq b_{\max }, c_{\min } \leq c(t) \leq c_{\max }$, where $u_{a}(t)$ is given in $(23)$, and

$$
u_{s}(t)=b(t)\left(\ddot{z}_{s}(t)-\ddot{z}_{u}(t)\right)+c(t)\left(\dot{z}_{s}(t)-\dot{z}_{u}(t)\right) .
$$

Denote $a(t)=\ddot{z}_{s}(t)-\ddot{z}_{u}(t)$ and $v(t)=\dot{z}_{s}(t)-\dot{z}_{u}(t)$, and

$$
\left\{\begin{array}{l}
u_{s \_ \text {max }}(t)=\max _{b(t), c(t)}\{a(t) b(t)+v(t) c(t)\}, \\
u_{s_{-} \min }(t)=\min _{b(t), c(t)}\{a(t) b(t)+v(t) c(t)\} .
\end{array}\right.
$$

Note that $u_{s \_ \text {max }}(t)$ and $u_{s \_ \text {min }}(t)$ represent the maximal and the minimal achievable forces of the semi-active suspension at time instant $t$, respectively. The explicit control law at each time instant can be obtained as

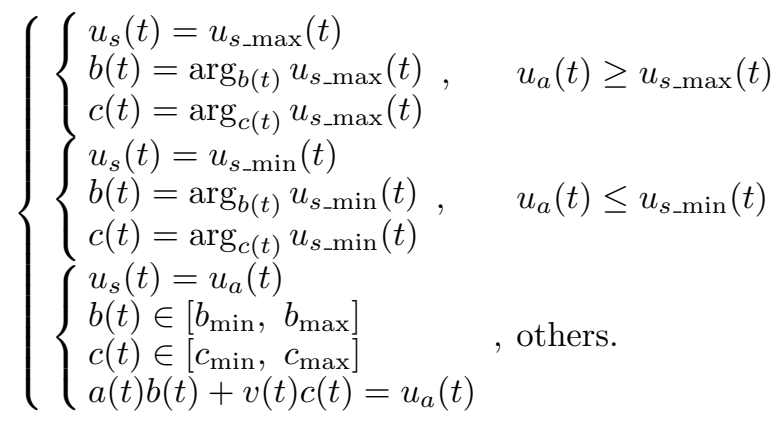

Here, $\arg _{b(t)} u_{s \_ \text {max }}(t)$ is interpreted as the inertance $b(t)$ at time $t$ that makes $u_{s}$ maximal, where similar interpretations can be done on $\arg _{c(t)} u_{s_{\_} \max }(t), \arg _{b(t)} u_{s_{-} \min }(t)$ and $\arg _{c(t)} u_{s_{-} \min }(t)$. The underlining idea of the explicit control law (26) is to saturate the active suspension force between the maximal and minimal achievable semi-active 
suspension forces $u_{s_{-} \max }(t)$ and $u_{s_{-} \min }(t)$. Since $u_{s}(t)$ is a linear function with respect to $b(t)$ and $c(t)$, both $b(t)$ and $c(t)$ for the first two cases in (26) are the combinations of the extreme values of the inertance and damping coefficient $\left(b_{\max }, b_{\min }, c_{\max }, c_{\min }\right)$.

For the third case in (26), the inertance $b(t)$ and damping coefficient $c(t)$ are obtained as

$$
\begin{aligned}
u_{c}(t)= & \frac{1}{2}\left(\max \left\{u_{c \_ \text {min }}, u_{a}(t)-u_{b_{\_} \max }\right\}\right. \\
& \left.+\min \left\{u_{c \_ \text {max }}, u_{a}(t)-u_{b_{-} \min }\right\}\right), \\
u_{b}(t)= & u_{a}(t)-v(t) c(t),
\end{aligned}
$$

where $u_{b}(t)=a(t) b(t)$ and $u_{c}(t)=v(t) c(t)$ are the forces generated by the semi-active inerter and the semi-active damper, respectively, and

$$
\begin{gathered}
\left\{\begin{array}{l}
u_{b_{-} \max }=\max \left\{a(t) b_{\max }, a(t) b_{\min }\right\}, \\
u_{b_{-} \min }=\min \left\{a(t) b_{\max }, a(t) b_{\min }\right\},
\end{array}\right. \\
\left\{\begin{array}{l}
u_{c_{-} \max }=\max \left\{v(t) c_{\max }, v(t) c_{\min }\right\}, \\
u_{c \text { min }}=\min \left\{v(t) c_{\max }, v(t) c_{\min }\right\} .
\end{array}\right.
\end{gathered}
$$

If $v(t) \neq 0$ and $a(t) \neq 0$, then $c(t)=u_{c}(t) / v(t)$ and $b(t)=u_{b}(t) / a(t)$. If either $v(t)$ or $a(t)$ is equal to zero, the damping coefficient or inertance is set to be the minimum value correspondingly.

Note that if only a semi-active damper is employed, that is, $b(t)=0$ all the time, the control law (26) reduces to the "clipped optimal" control for the conventional semi-active suspension. Accordingly, if $c(t)=0$ all the time, that is, only a semi-active inerter is employed, then a sub-optimal control law can be derived similarly by saturating $u_{a}(t)$ between $u_{b_{-} \min }$ and $u_{b_{-} \max }$.

Now, three semi-active suspension schemes have been derived for three cases based on whether a semi-active inerter and a semi-active damper are employed or not, as summarized in Table 1.

Table 1. Semi-active suspension schemes.

\begin{tabular}{lcc}
\hline Scheme & Semi-active damper & Semi-active inerter \\
\hline Semi-I \& D & Employed & Employed \\
Semi-I & Not employed & Employed \\
Semi-D & Employed & Not employed \\
\hline
\end{tabular}

Remark 2. It can be checked that for each time instant $t$, the following inequalities

and

$$
\left[u_{c \_ \text {min }}(t), u_{c \_ \text {max }}(t)\right] \subseteq\left[u_{s \_ \text {min }}(t), u_{s \_\max }(t)\right],
$$

$$
\left[u_{b \_\min }(t), u_{b \_\max }(t)\right] \subseteq\left[u_{s \_\min }(t), u_{s \_\max }(t)\right],
$$

hold by carefully choosing $c(t)$ and $b(t)$, respectively. This means that for the same target active control law, the suspension force of Semi-I \& D suspension are much more likely to be equal to the target active force than Semi-I and Semi-D suspensions do. Hence, the Semi-I \& D suspension has the potential to improve suspension performance in comparison with the other two suspensions, which will be demonstrated in the following sections.

\section{SIMULATION RESULT}

In this section, numerical simulation is carried out based on a quarter-car model of a passenger sedan in (Rajamani
(2005)), the parameters of which are shown in Table 2. The weighting factors in $(20)$ are $\rho_{1}=1, \rho_{2}=1000$, $\rho_{3}=100000, r=1 \times 10^{-6}$. Two types of road profiles will be employed to test the performance of the proposed semi-active suspension. For the parameters in Table 2 and weighting factors in (20), the passive suspension with the optimal passive damping coefficient $2110.92 \mathrm{Ns} / \mathrm{m}$ is employed for comparison.

\section{Table 2. Vehicle parameters (Rajamani} (2005)).

\begin{tabular}{lc}
\hline Parameter & Value \\
\hline Sprung mass, $m_{s}$ & $250 \mathrm{~kg}$ \\
Unsprung mass, $m_{u}$ & $45 \mathrm{~kg}$ \\
Static stiffness, $k_{s}$ & $16 \mathrm{kN} / \mathrm{m}$ \\
Tyre stiffness, $k_{t}$ & $160 \mathrm{kN} / \mathrm{m}$ \\
\hline
\end{tabular}

\subsection{Bump test}

A single bump taken from (Hać (1992)) is described below

$$
z_{r}(t)=\left\{\begin{array}{cc}
c(1-\cos 20 \pi(t-0.1)), & \text { for } t \in[0.1,0.2] \\
0 & \text { otherwise }
\end{array}\right.
$$

where $2 c$ and $t$ are the height of the bump in $[\mathrm{m}]$ and time in [sec], respectively, with $2 c=0.1 \mathrm{~m}$. The parameters of the semi-active damper and semi-active inerter are $c_{\min }=0 \mathrm{Ns} / \mathrm{m}, c_{\max }=3000 \mathrm{Ns} / \mathrm{m}, b_{\min }=0 \mathrm{~kg}$ and $b_{\max }=100 \mathrm{~kg}$.

The simulation results are shown in Fig. 2, where the lines of the Semi-I \& D suspension match those of active suspension much better than those of other suspensions do. Especially, it is clearly shown in Fig. 2(d) that the Semi-I \& D suspension best approximates the active force, which is consistent with Remark 2. Semi-I suspension performs poorly because there is no damping element in the Semi-I suspension to dissipate the energy.

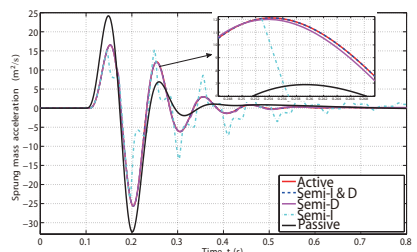

(a)

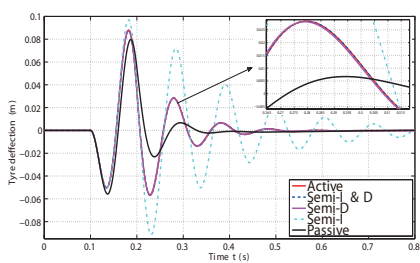

(c)

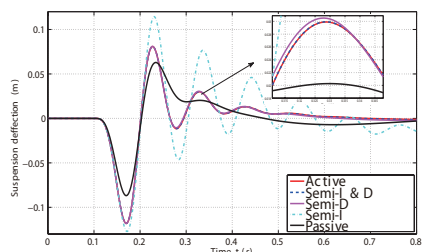

(b)

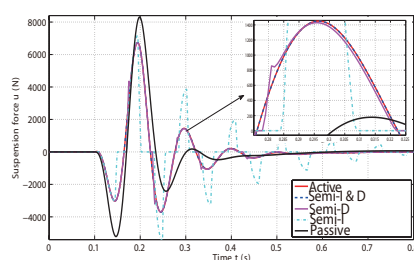

(d)
Fig. 2. Bump test. (a) Sprung mass acceleration; (b) Suspension deflection; (c) Tyre deflection; (d) Suspension force.

\subsection{Random road profile test}

Typical random road profiles can be described with spectral density as 
Table 3. Road description classified by ISO 8608 (Tyan et al. (2009)).

\begin{tabular}{|c|c|c|c|}
\hline Road Class & $\sigma\left(10^{-3} \mathrm{~m}\right)$ & $\Phi\left(\Omega_{0}\right)\left(10^{-6} \mathrm{~m}^{3}\right), \Omega_{0}=1$ & $\alpha(\mathrm{rad} / \mathrm{m})$ \\
\hline A (very good) & 2 & 1 & 0.127 \\
\hline B (good) & 4 & 4 & 0.127 \\
\hline C (average) & 8 & 16 & 0.127 \\
\hline $\mathrm{D}$ (poor) & 16 & 64 & 0.127 \\
\hline $\mathrm{E}$ (very poor) & 32 & 256 & 0.127 \\
\hline
\end{tabular}

$$
\Phi(\Omega)=\Phi\left(\Omega_{0}\right)\left(\frac{\Omega}{\Omega_{0}}\right)^{-\omega},
$$

where $\Omega$ is the wave number with unit $[\mathrm{rad} / \mathrm{m}]$ and $\Phi_{0}=$ $\Phi\left(\Omega_{0}\right)$ in $\left[\mathrm{m}^{2} /(\mathrm{rad} / \mathrm{m})\right]$ is the value of the Power Spectral Density (PSD) at $\Omega_{0}=1 \mathrm{rad} / \mathrm{m}$. $\omega$ denotes the waviness, where $\omega=2$ for most of the road surface. As suggested by the International Organization for Standardization (ISO) (ISO8608 (1995)), typical road profiles based on the value of $\Phi_{0}$ are formulated denoted as Class A to Class $\mathrm{E}$ as shown in Table 3. Each road profile can be realized by using the shaping filter method (Tyan et al. (2009)) as descried below:

$$
\dot{z}_{r}(t)=-\alpha V z_{r}(t)+w(t)
$$

where the white noise process $w(t)$ with the spectral density $\Psi_{w}=2 \alpha V \sigma^{2}$ is assumed to pass through a linear filter and $V$ is the vehicle forward speed. The correspondence between the parameters $\sigma$ and $\alpha$ in the linear filter and the ISO standard are shown in Table 3 (see (Tyan et al. (2009)) for details).

For the random road profile, the following quantitative performance indexes are defined to represent ride comfort, suspension working space and road holding, respectively:

$$
\begin{aligned}
J_{a c c} & =\sqrt{\frac{1}{T} \int_{0}^{T} \ddot{z}_{s}^{2} d t}, \\
J_{s w s} & =\sqrt{\frac{1}{T} \int_{0}^{T}\left(z_{s}-z_{u}\right)^{2} d t}, \\
J_{r h d} & =\sqrt{\frac{1}{T} \int_{0}^{T}\left(z_{u}-z_{r}\right)^{2} d t},
\end{aligned}
$$

where $T$ is the simulation time. To reflect the total performance $J$ in (20), the root mean value of $J(R M . J)$ is defined as

$$
R M . J=\sqrt{\frac{1}{T} \int_{0}^{T} \rho_{1} \ddot{z}_{s}^{2}+\rho_{2}\left(z_{u}-z_{r}\right)^{2}+\rho_{3}\left(z_{u}-z_{r}\right)^{2} d t} .
$$

To reflect the tracing ability of these three semi-active suspensions, the RMS force tracking error $u_{e}$ is defined as

$$
u_{e}=\sqrt{\frac{1}{T} \int_{0}^{T}\left(u_{s}-u_{a}\right)^{2} d t} .
$$

In this paper, the simulation time $T=50 \mathrm{~s}$ is used and the Class B and Class D roads are employed for the following simulation. The results are shown in Table 4. It is clear

\begin{tabular}{|c|c|c|c|c|c|c|}
\hline Road & Performance & Active & Semi-I \& D & Semi-D & Semi-I & Passive \\
\hline \multirow{5}{*}{ B road } & $R M . J\left(\times 10^{-1}\right)$ & 6.55 & 6.56 & 6.59 & 7.52 & 7.37 \\
\hline & $J_{a c c}\left(\mathrm{~m} / \mathrm{s}^{2}\right)$ & 0.41 & 0.41 & 0.42 & 0.36 & 0.57 \\
\hline & $J_{s w s}(\mathrm{~mm})$ & 3.11 & 3.12 & 3.16 & 4.28 & 2.69 \\
\hline & $J_{r h d}(m m)$ & 1.58 & 1.57 & 1.57 & 2.05 & 1.44 \\
\hline & $u_{e}(\mathrm{~N})$ & 0 & 8.18 & 17.16 & 77.24 & 65.64 \\
\hline \multirow{5}{*}{$\mathrm{D}$ road } & $R M . J$ & 2.62 & 2.62 & 2.64 & 3.01 & 2.95 \\
\hline & $J_{a c c}\left(\mathrm{~m} / \mathrm{s}^{2}\right)$ & 1.65 & 1.65 & 1.69 & 1.43 & 2.29 \\
\hline & $J_{s w s}(\mathrm{~cm})$ & 1.24 & 1.25 & 1.26 & 1.71 & 1.07 \\
\hline & $J_{r h d}(\mathrm{~mm})$ & 6.32 & 6.31 & 6.27 & 8.19 & 5.78 \\
\hline & $u_{e}(\mathrm{~N})$ & 0 & 32.71 & 68.62 & 308.96 & 262.56 \\
\hline
\end{tabular}
that Semi-I \& D suspension performs better than both Semi-D and Semi-I suspensions, which effectively demonstrates the benefits of the proposed semi-active inerter. Table 4 shows that ride comfort and suspension working
Table 4. The simulation result with random road profile.

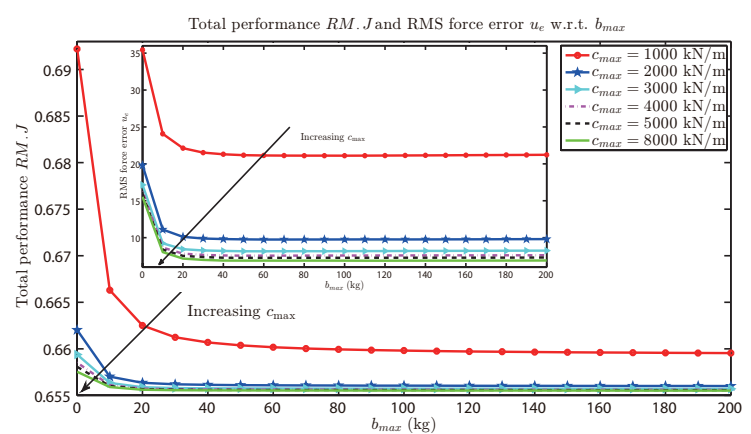

Fig. 3. Total performance $J$ and RMS force tracking error $u_{e}$ with respect to different $c_{\max }$ and $b_{\max }$. The inner figure is the RMS force tracking error $u_{e}$ w.r.t. $c_{\max }$ and $b_{\max }$; The outer figure is the total performance $J$ w.r.t. $c_{\max }$ and $b_{\max }$.

space are improved by using semi-active inerter, in the meanwhile almost equal road holding performance with active suspension is obtained. The road holding performance of Semi-D suspension is slightly better than the active and Semi-I \& D suspension due to the compromise between these performance objectives. Considering the RMS tracking error $u_{e}$ in Table 4 , it is clear that the tracking error of Semi-I \& D suspension is much smaller than those of Semi-D and Semi-I suspensions, which is consistent with Remark 2.

To investigate the influence of the upper bounds of inertance and damping coefficient $b_{\max }$ and $c_{\max }$ on the performance of Semi-I \& D suspension, the Semi-I \& D suspension is simulated with different $b_{\max }$ and $c_{\max }$ for Class B road profile. Fig. 3 shows that the total performance as well as the force error can be effectively decreased by increasing $b_{\max }$ and $c_{\max }$. It is also shown in Fig. 3 that although significant improvements are obtained by Semi-I \& D suspension, it cannot match the active one perfectly, because of the semi-active nature of Semi-I \& D suspension.

\section{CONCLUSIONS}

This paper has investigated the application of semi-active inerter in semi-active suspension. The concept of semiactive inerter has been defined based on the concept of passive inerter. Meanwhile, a force-tracking method to design semi-active suspension with a semi-active inerter and a semi-active damper has also been proposed. In the first part of this paper, the optimal solution with respect 
to a general performance index for LQR control in the RSS framework was derived. It was shown that by using the general performance index in the RSS framework, it is possible to design optimal controller in the RSS framework, which is equivalent to the one in the SS framework. In the second part, a force-tracking algorithm was derived to adjust inertance and damping coefficients of the semi-active inerters and the semi-active dampers. Numerical simulations demonstrated that the semi-active suspension with a semi-active inerter and a semi-active damper can approximate the target active control force much better than the conventional strut (which only contains a semi-active damper) does. As a result, better performance was obtained, which effectively demonstrated the necessity and benefit of employing semi-active inerter in vehicle suspension. In addition, since only accelerations and suspension forces were measured in the proposed semiactive suspension, the noise amplification issue caused by differentiation was avoided and it was easy to implement the proposed method in practice.

\section{REFERENCES}

Chen, M. Z. Q., Papageorgiou, C., Scheibe, F., Wang, F. C., \& Smith, M. C. (2009). The missing mechanical circuit element, IEEE Circ. Syst. Mag., 9(1), 10-26.

Chen, M. Z. Q., Hu, Y., \& Du, B. (2012). Suspension performance with one damper and one inerter. the 24th Chinese Control and Decision Conference, Taiyuan, China (pp. 3551-3556).

Chen, M. Z. Q., Hu, Y., Huang, L., \& Chen, G. (2014). Influence of inerter on natural frequencies of vibration systems. Journal of Sound and Vibration, 333 (7), 18741887.

Chen, M. Z. Q. \& Smith, M. C. (2008). Electrical and mechanical passive network synthesis, in Recent Advances in Learning and Control, New York: Springer-Verlag, 71, $35-50$.

Chen, M. Z. Q. \& Smith, M. C. (2009a). A note on tests for positive-real functions. IEEE Trans. on Automatic Control, 54(2), 390-393.

Chen, M. Z. Q. \& Smith, M. C. (2009b). Restricted complexity network realizations for passive mechanical control. IEEE Trans. on Automatic Control, 54(10), 2290-2301.

Chen, M. Z. Q., Wang, K., Zou, Y., \& Lam, J. (2013). Realization of a special class of admittances with one damper and one inerter for mechanical control. IEEE Trans. Automatic Control, 58(7), 1841-1846.

Du, H. \& Zhang, N. (2007). $\mathrm{H}_{\infty}$ control of active vehicle suspensions with actuator time delay. Journal of Sound and Vibration, 301(1), 236-252.

Du, H., Sze, K. Y., \& Lam, J. (2005). Semi-active $H_{\infty}$ control of vehicle suspension with magneto-rheological dampers. Journal of Sound and Vibration, 283, 981-996.

Du, H. \& Zhang, N. (2009). Fuzzy control for nonlinear uncertain electrohydraulic active suspensions with input constraint. IEEE Transactions on Fuzzy Systems, 17(2), $343-356$

Duan, Y. F., Ni, Y. Q., \& Ko, J. M. (2005). Statederivative feedback control of cable vibration using semiactive magnetorheological dampers. Computer-Aided Civil and Infrastructure Engineering, 20(6), 431-449.
Gao, H., Sun, W., \& Shi, P. (2010). Robust sampled-data $\mathrm{H}_{\infty}$ control for vehicle active suspension systems. IEEE Transactions on Control Systems Technology, 18(1), 238-245.

Gao, H., Lam, J., and Wang, C. (2006). Multi-objective control of vehicle active suspension systems via loaddependent controllers. Journal of Sound and Vibration, 290(3), 654-675.

Hu, Y., Li, C., \& Chen, M. Z. Q. (2012). Optimal control for semi-active suspension with inerter. the 31st Chinese Control Conference, Hefei, China (pp. 2301-2306).

Hać, A. (1992). Optimal linear preview control of active vehicle suspension. Vehicle System Dynamics, 21(1), 167-195.

ISO 8608:1995-09-01, Mechanical Vibration-Road Surface Profiles-Reporting of Measured Data, International Organization for Standardization (ISO).

Kwak, S. K., Washington, G., \& Yedavalli, R. K. (2002). Acceleration-based vibration control of distributed parameter systems using the reciprocal state-space framework. Journal of Sound and Vibration, 251(3), 543-557.

Li, H., Liu, H., Gao, H., and Shi, P. (2012). Reliable fuzzy control for active suspension systems with actuator delay and fault. IEEE Transactions on Fuzzy Systems, $20(2), 342-357$.

Rajamani, R. (2005). Vehicle Dynamics and Control. Springer-Verlag, New York.

Smith, M. C. (2002). Synthesis of mechanical networks: The inerter. IEEE Trans. on Automatic Control, 47(1), 1648-1662.

Smith, M. C. \& Wang, F. C. (2004). Performance benefits in passive vehicle suspensions employing inerters. Veh. Syst. Dyn., 42(4), 235-257.

Savaresi, S. M., Poussot-Vassal, C., Spelta, C., Sename, O., \& Dugard, L. (2010). Semi-Active Suspension Control Design for Vehicles. Elsevier, Butterworth Heinemann.

Tseng, Y. W. (1997). Control Design of Linear Dynamic Systems with Matrix Differential Equations for Aerospace Applications, Ph.D. Thesis, Department of Aerospace Engineering, The Ohio State University, USA.

Tyan, F., Hong, Y. F., Tu, S. H., \& Jeng, W. S. (2009). Generation of random road profiles. Journal of Advanced Engineering, 1373-1378.

Wang, K., Chen, M. Z. Q., \& Hu, Y. (2014). Synthesis of biquadratic impedances with at most four passive elements. Journal of the Franklin Institute, 351 (3), 1251-1267.

Wang, K. \& Chen, M. Z. Q. (2012). Generalized seriesparallel RLC synthesis without minimization for biquadratic impedances. IEEE Trans. on Circuits and Systems II: Express Briefs, 59 (11), 766-770.

Zhou, K., Doyle, J., \& Glover, K. (1996). Robust and Optimal Control, Prentice Hall, NJ. 